\title{
Interchange Sight Distance and Design: Aspects and Implementation
}

\author{
Shy Bassan \\ Amy Metom Engineers \& Consultants, Ltd., Tel Aviv, Israel \\ Email: bassans@ netvision.net.il
}

How to cite this paper: Bassan, S. (2021) Interchange Sight Distance and Design: Aspects and Implementation. Open Journal of Civil Engineering, 11, 358-377. https://doi.org/10.4236/ojce.2021.113021

Received: July 21, 2021

Accepted: September 27, 2021

Published: September 30, 2021

Copyright (c) 2021 by author(s) and Scientific Research Publishing Inc. This work is licensed under the Creative Commons Attribution International License (CC BY 4.0).

http://creativecommons.org/licenses/by/4.0/

\begin{abstract}
The primary purpose of an interchange is to enable an appropriate level of access to the freeway in a safe and beneficial way to the driver. It improves road safety and traffic operation by reducing or eliminating traffic conflicts that would occur if grade separation were not provided (e.g. intersection). Crossing conflicts are eliminated and turning conflicts are either eliminated or minimized. The paper focuses on sight distance criteria in the geometric design of interchanges and junctions, specifically, implementation of stopping sight distance (SSD) and decision sight distance (DSD). These issues incorporate implementation of sight distance policy for: ramps' geometric design, ramp terminals' components, and basic lanes' reduction. These sight distance criteria can alleviate safety by reducing conflicts and confusion to the driver and by keeping alignment simple, direct, and consistent. Specifically, DSD affects traffic control and intelligent transportation systems applications.
\end{abstract}

\section{Keywords}

Interchange, Sight Distance, Decision, Ramp, Terminal

\section{Introduction}

An interchange is a system of interconnecting highways in combination with one or more grade separations that provides the movements of traffic flow between two or more highways on different levels. At least one of the interconnecting highways is a full access control highway (freeway). Crossing conflicts are eliminated by grade separation and turning conflicts are minimized depending on interchange functional classification and configuration. The driver is required to select a suitable speed, accelerate and decelerate, and choose appropriate lanes in order to make merging and diverging maneuvers.

The basic principles of interchange design assist the driver to pass with mini- 
mum disturbance of exiting and entering traffic without difficulty that the through highway is the correct one for the driver's destination. A high quality design enables the driver to understand the operation of the interchange without being misled, or surprised by any design feature. Route continuity, provision of adequate sight distance, and uniformity of signing are important features in this manner.

Specific literature review related to sight distance utilization on the topics of entrance ramp terminals and exit ramp terminals is embedded in Sections 4.1.1 and 4.2.1 correspondingly. These sections include relevant examples from Australia and Canada for comparison purpose to the recommended implementation of decision sight distance on ramp terminals. Canada [1] [2] proposes comparatively higher ranges of decision sight distance design values in order to gain a view of the entire speed-change lane (acceleration lane or deceleration lane) at the bull-nose. Australia [3] proposes 4 seconds of travel by the merging driver along the acceleration lane (Section 4.1.1), and a two-stage maneuver procedure to search the start of exit taper and for the remaining alignment of the "exit lane" and exit nose position (Section 4.2.1).

\subsection{Safety Background}

The safety level of an interchange is better than the safety level of an intersection for similar traffic volumes, while the intersection still operates well without delays. An intersection can have a potential of traffic collisions, specifically side-head or side/angle collisions.

An intersection might be converted to an interchange in order to alleviate safety problems related to high speed on the intersecting highways (specifically before isolated intersections), insufficient gaps, sight distance restrictions, difficult turns, and restrictions of vertical and horizontal alignments near the intersection. When the intersection experiences severe accidents, the first stage for improvements is the implementation of local and focused geometric solutions with minimal cost. If these solutions are not useful for improving traffic safety, then grade separation or a compact grade-separated junction (CGSJ) or an interchange might be justified.

The vital elements of interchange safety refer to interchange configurations, traffic controls and spacing. Safety research generally focused on most common interchange types: diamonds and cloverleafs [4]. Within the interchange individual components safety research concentrated on ramps, ramp terminals, speed change lanes, alignment and spacing. Ramp safety elements are acceleration and deceleration lanes, weaving sections, ramp alignment, and ramp terminals. The interchange alignment factors include sight distance which is discussed in this study, vertical and horizontal curves which depend on sight distance policy and grades.

Figure 1 presents a schematic diamond interchange with its individual components. Ramps, acceleration lanes, and crossroad ramp terminals are considered as individual elements [5]. 
Mainline freeways (MF)

Interchange ramps $(\mathrm{R})$

Acceleration lanes (AL)

Crossroad ramp terminals (RT)

Crossroad roadway segments (RS)

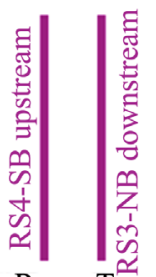

(North Ramp Terminal)

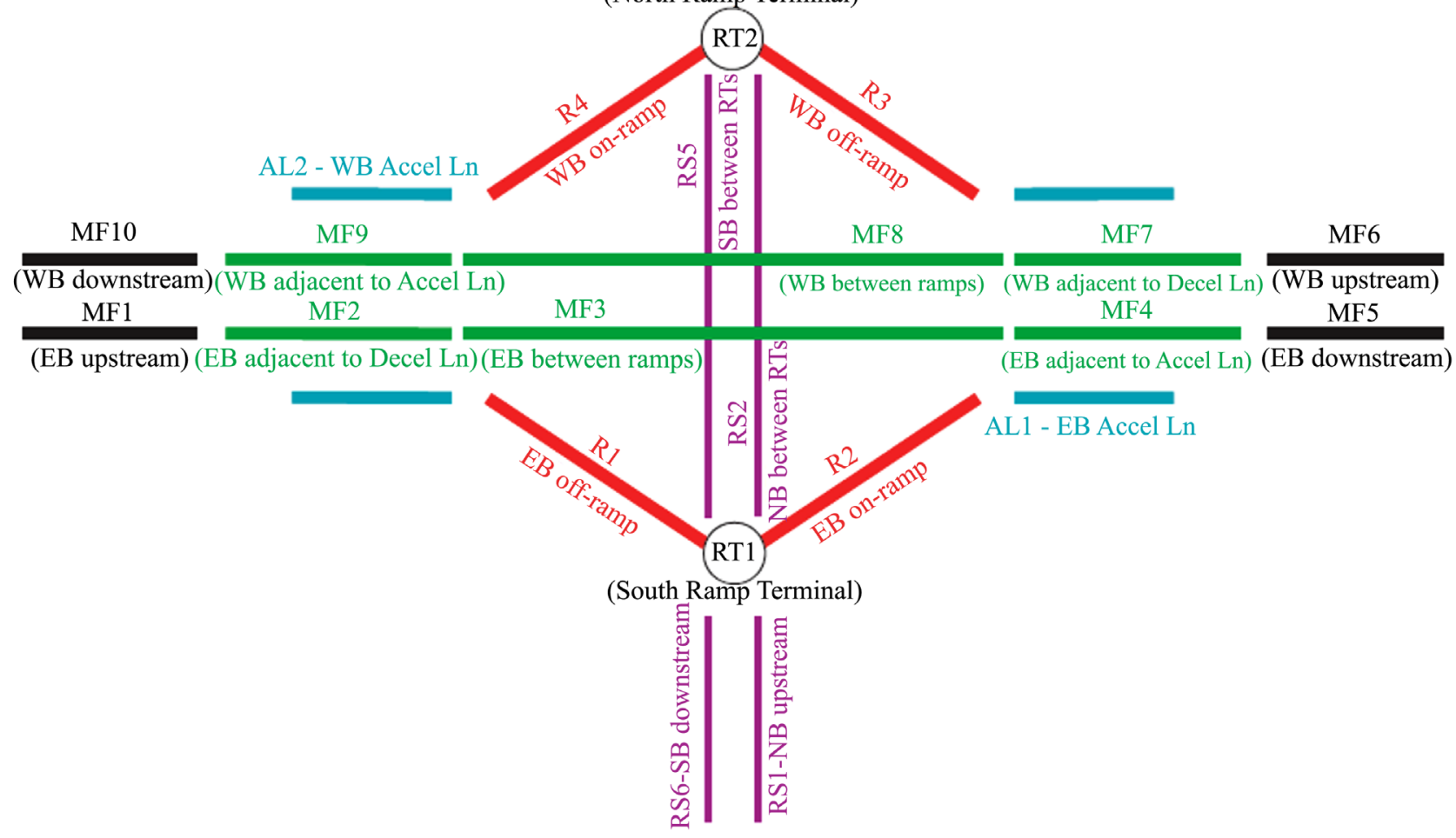

Figure 1. Interchange illustrating individual components [5].

Twomey et al. [4] concluded that it is safer to merge or diverge a given traffic volume of vehicles with or from a freeway at several minor flow ramps (CD road etc.) than at single high volume on-ramps and off-ramps.

Overall, designers can reduce drivers' pressure at interchanges by keeping alignment simple, direct, and consistent, and by providing sight distance greater than the stopping sight distance (i.e. decision sight distance) which results in employing above minimum design criteria for interchange geometric elements [1].

\subsection{Interchange Sight Distance Elements and Implementation}

The paper introduces implementation of sight distance policy for: ramps' geometric design, ramp terminals' components, and basic lanes' reduction. These implementations of sight distance types (including their relevant models and parameters), for interchange design, especially on ramps, ramp terminal influence zones (along acceleration lane and prior to exit ramp along the major highway), and basic lanes' reduction prior to or after two lane exit ramp terminals are not available in the literature. 
Section 2 introduces a unique decision sight distance model to be implemented in: 1) ramp design when the ramp is connected to the minor intersecting highway by a signalized intersection with additive lanes (presented in Section 3); 2) prior to interchange ramp terminal zone along the main lanes, and along the acceleration lane merging to the highway (presented in Section 4). Surplus practical applications of decision sight distance are relevant to the design of basic lane reduction along freeways and interchanged highways. These applications are documented in Section 5. The final part of Section 2 includes practical implementations of stopping sight distance, specifically for ramp design when the ramp is connected to the minor intersecting highway by an intersection (unsignalized, roundabout, or signalized), without additive lanes (presented in Section 3).

\section{Sight Distance Principal Policy and Models}

Decision sight distance (DSD) is defined as the distance at which drivers can detect a hazard or signal in a cluttered roadway environment, recognize it (or its threat potential), select the appropriate speed and path, and perform the required action safely and efficiently [6].

The basic sight distance for freeway geometric design is decision sight distance (DSD). DSD correlates the sighting focused range of the driver and his expectancies. It enables the driver:

- Feel the general direction of travel and driving alignment.

- Be ready to perform safely all maneuver options required e.g. accelerating, decelerating, changing lanes, and turnings.

The decision sight distance (DSD) enables a maneuver which is less risky than the braking maneuver of stopping sight distance until a complete stop. The driver should be able to choose the speed and the suitable path for the specific maneuver required and correct an erroneous maneuver action. This distance is usually suitable when drivers must make complex decisions, when information is difficult to find or is unusual, and when unusual maneuvers are required.

The DSD might be implemented before critical points of the road alignment such as un-signalized intersections, interchanges (merging and diverging ramps), acceleration and deceleration lanes, weaving zones, abrupt changes in the alignment profile, and warning or guidance areas. The Israeli DSD model [7] consists of three driving maneuvers stages:

1) The pre-maneuver stage.

2) The braking action from the free flow speed (the design speed for the purpose of highway design) to the maneuver speed.

3) The maneuver operation.

The representative pre-maneuver time consists of 2 seconds for perception and recognition and 3.5 seconds for deciding the maneuver solution. Therefore, the total pre-maneuver time is 5.5 seconds and does not depend on the design speed. This pre-maneuver time value is based on the lower bound of McGee's [8] 
"hazard avoidance model" (including field work that was performed to operationally validate the DSD model results) which does not include the second phase of the Israeli model (braking action).

The maneuver time is the time it takes the driver to fully complete the maneuver (lane change, bypassing or escaping from the hazard, detours or construction areas, exit lane drop, etc.). It involves a change in path and/or speed depending upon the nature of the hazard. Change in path for example signifies that a lane change would be the selected maneuver. A typical maneuvering time range is 3.5 - 4.5 seconds (based on McGee [6] and AASHTO [9] [10]), even though McGee's range for generating DSD design values is $4-4.5$ seconds. The upper limit corresponds to the lowest design speed and the lower limit is appropriate with the highest design speed. The intermediate values of maneuver time (in the Israeli model) were generated by linear interpolation as introduced in Table 1.

The maneuver velocities $\left(V_{M}\right)$ are lower than the design speed by $10-60$ $\mathrm{km} /$ hour. As the design speed increases the algebraic difference between $V_{d}$ and $V_{M}$ increases as well. These velocities are based on empirical evidence gained in Australia [11], where the second and third phase of the proposed DSD model, are unified to one phase.

DSD Model Equation

The DSD proposed model is introduced in Equation (1).

$$
\mathrm{DSD}=\frac{5.5}{3.6} \cdot V_{D}+\frac{V_{D}^{2}-V_{M}^{2}}{2 \cdot 3.6^{2} \cdot d}+\frac{T_{M}}{3.6} \cdot V_{M}=1.53 V_{D}+\frac{V_{D}^{2}-V_{M}^{2}}{25.92 \cdot d}+\frac{T_{M}}{3.6} \cdot V_{M}
$$

DSD—decision sight distance (m);

$V_{D}$-design speed (km/hour);

$d$-average deceleration rate $\left(\mathrm{m} / \mathrm{sec}^{2}\right)$, as applied for SSD (design values of Table 3);

$T_{M}$-maneuver time (sec);

$V_{M}$-average maneuver speed.

Table 1. Decision sight distance parameters, and design values.

\begin{tabular}{|c|c|c|c|c|c|c|c|c|c|c|c|c|}
\hline Design speed $(\mathrm{km} / \mathrm{hr})$ & 30 & 40 & 50 & 60 & 70 & 80 & 90 & 100 & 110 & 120 & 130 & 140 \\
\hline Maneuver speed $(\mathrm{km} / \mathrm{hr})$ & 25 & 30 & 35 & 40 & 50 & 50 & 60 & 60 & 70 & 80 & 80 & 80 \\
\hline Deceleration $\left(\mathrm{m} / \mathrm{sec}^{2}\right)^{(1)}$ & 4.3 & 4.3 & 4.3 & 4.3 & 4.2 & 4.1 & 4.0 & 3.9 & 3.8 & 3.7 & 3.7 & 3.7 \\
\hline Maneuver time (sec) & 4.5 & 4.5 & 4.39 & 4.28 & 4.17 & 4.06 & 3.94 & 3.83 & 3.72 & 3.61 & 3.50 & 3.50 \\
\hline DSD design values (m) & 80 & 110 & 135 & 160 & 190 & 220 & 255 & 290 & 325 & 360 & 400 & 440 \\
\hline DSD type C (AASHTO [9]) $)^{(2)}$ & 85 & 115 & 145 & 170 & 200 & 230 & 270 & 315 & 330 & 360 & 390 & 420 \\
\hline DSD type D (AASHTO [9]) & 95 & 125 & 170 & 205 & 235 & 270 & 315 & 355 & 380 & 415 & 450 & 485 \\
\hline DSD: McGee [8] & - & $120-160$ & - & $170-230$ & - & $230-310$ & - & $310-400$ & - & $360-470$ & - & $420-540$ \\
\hline
\end{tabular}

(1)Deceleration rates were adopted from SSD; (2)Maneuver C: speed, path, direction change on rural roadway; ${ }^{(3)}$ Maneuver D: speed, path, direction change on suburban roadway. 
Table 1 presents the Israeli DSD model design parameters and its resulted design values. Also included in Table 1 are DSD values proposed by AASHTO [9] [10] and by McGee [8]. These values refer to an avoidance maneuver where the pre maneuver time is larger than the brake reaction time, to provide the driver additional time to detect and recognize the roadway or traffic situation, identify alternative maneuvers, and initiate a response at critical locations. The supplemented design values refer to AASHTO avoidance maneuver types $\mathrm{C}$ (rural road) or D (suburban road): speed or path or direction change without a braking component for rural road and suburban road respectively. These maneuver types do not include a braking component but do include an increased fixed value of pre maneuver plus maneuver time. This fixed value (pre maneuver time plus maneuver time) ranges between 10.2 seconds to 11.2 seconds for maneuver type $\mathrm{C}$ and between 12.1 to 12.9 for maneuver type D, based on AASHTO [9]. On rural roadways the maneuver process is assumed to be faster than in suburban roads where the driving situation might generate more conflicts to the driver. The DSD proposed values based on Israeli model (Equation (1)) are slightly shorter but similar to maneuver type C, applied for rural roadways in the US [9].

\subsection{Implementation of Decision Sight Distance (DSD)}

Decision sight distance is measured from the driver's eye height of $1.05 \mathrm{~m}$ to an object height of $0.6 \mathrm{~m}$. It is basically implemented prior to the interchange ramp terminal zone along the main lanes of fully interchanged highway or along the acceleration lane merging to the highway from the ramp shorter object values should be considered $(0.15 \mathrm{~m}$ or $0.0 \mathrm{~m})$.

\section{Typical scenarios for implementation of DSD:}

- Along freeways' alignment.

- On fully interchanged highways, while approaching the exit ramp.

- Along the ramp in order to notice the traffic regulations and the interchange configuration prior to merging to the major highway.

- Along the ramp, approaching a signalized intersection if there is a change in lane configuration in the intersection leg.

\subsection{Implementation of Stopping Sight Distance (SSD)}

SSD should be implemented along ramps prior to an intersection without increasing the number of lanes in the intersection approach.

Sight distance restrictions by vertical and horizontal curves along the ramp alignment, and also by bridge structures along the interchange zone should be considered.

\section{Sight Distance Implementation for Ramps' Geometric Design}

The sight distance types for ramp design can be stopping sight distance (SSD) or decision sight distance (DSD). Unique implementation of SSD and DSD for 
ramp design does not appear in specific literature sources. In general SSD should be implemented for all ramps with similar principles for major roads and minor roads (Austroads [3] "interchanges"). Approach sight distance (ASD) can be implemented when approaching at-grade intersections at ramp terminals [3]. This design feature which is composed of reaction time (1.5 - 2.5 seconds) and braking is similar to SSD used for major roads and minor roads.

However, sight distance at exit and entrance ramp terminals should be longer than SSD [1] [2] [3] in order to allow for direction-finding as well as operational decision. These issues are discussed in Section 4. The Israeli policy differentiates between implementation of SSD and DSD on ramps as follows:

\subsection{Implementation of Stopping Sight Distance for Ramp Design}

SSD is the minimum sight distance that should be maintained along the ramp alignment. The SSD needs to be adjusted to longitudinal grade and ramp design speed.

Ramps that are connected to the minor intersecting highway of an interchange by an unsignalized intersection without additive lanes in the intersection approach shall be designed according to SSD. Since the driver is more alerted while driving along the ramp (compared to the interurban section) the SSD could be calculated in accordance with PRT (perception reaction time) of 2.0 seconds.

The object height for designing a crest vertical curve along the approach to an unsignalized intersection (without additive lanes) is $0.15 \mathrm{~m}$.

The SSD model which is prevalent in many highway design guidelines (e.g. AASHTO [10], TAC [1], Austroads [3]) is presented in Equation (2). The stopping sight distance model has two components: 1) the distance traveled during the driver's reaction time; 2$)$ the distance traveled during braking by implementing equivalent deceleration rate $\left(d\right.$, meter $\left./ \mathrm{sec}^{2}\right)$.

The differences in SSD outcome are caused by its purpose and derived parameters.

$$
\mathrm{SSD}=\frac{t_{R}}{3.6} \cdot V_{d}+\frac{V_{d}^{2}}{2 \cdot 3.6^{2} \cdot d}
$$

where:

SSD—minimum stopping sight distance (m);

$V_{d}$-design speed $(\mathrm{km} / \mathrm{hr})$;

$d$-deceleration of passenger cars $\left(\mathrm{m} / \mathrm{s}^{2}\right)($ Table 2$)$;

$t_{R}$ (or PRT)-perception reaction time (s), 2.0 seconds for ramps and ramp terminal applications.

Typical SSD design values (which depend on the ramp design speed) are presented in Table 2.

\subsection{Implementation of Decision Sight Distance for Ramp Design}

DSD is the basic sight distance for the design of intersecting highways at the 
Table 2. Design values of SSD and DSD for passenger cars for ramp design leading to an intersection on the interchange minor roadway (level terrain: longitudinal grade smaller than $3 \%)$.

\begin{tabular}{ccccc}
\hline $\begin{array}{c}\text { Ramp design speed } \\
(\mathrm{km} / \text { hour })\end{array}$ & $\begin{array}{c}\text { Deceleration } \\
\left(\mathrm{m} / \mathrm{sec}^{2}\right)\end{array}$ & $\begin{array}{c}\text { Equivalent friction } \\
\text { coefficient }(\mathrm{a} / \mathrm{g})\end{array}$ & $\begin{array}{c}\text { SSD } \\
(\mathrm{m})^{(1)}\end{array}$ & $\begin{array}{c}\text { DSD } \\
(\mathrm{m})\end{array}$ \\
\hline 30 & 4.19 & 0.43 & 25 & 80 \\
40 & 4.19 & 0.43 & 40 & 110 \\
50 & 4.19 & 0.43 & 55 & 135 \\
60 & 4.19 & 0.43 & 70 & 160 \\
70 & 3.96 & 0.40 & 90 & 190 \\
80 & 3.76 & 0.38 & 115 & 220 \\
90 & 3.57 & 0.36 & 140 & 255 \\
100 & 3.41 & 0.35 & 170 & 290 \\
\hline
\end{tabular}

(1)PRT of 2 section for SSD calculation on ramps. Design values of DSD are based on Israeli DSD model (Table 1, Bassan [7] [12]).

interchange. Ramps that are connected to the minor intersecting highway of an interchange by a signalized intersection with additive lanes in the intersection approach shall be designed according to DSD. This situation is usually suitable for a ramp exiting from the major highway of the interchange and intersecting urban arterial (minor roadway of the interchange).

The object height for designing a crest vertical curve along the approach to a signalized intersection (with additive lanes) is $0.15 \mathrm{~m}$. Typical DSD design values (which depend on the ramp design speed) are also included in Table 2, based on Israel model documented in Section 3.

\subsection{Analysis of Vertical Curves on Ramps}

The analysis of vertical curves on ramps towards the intersection of interchange minor road is accomplished for either crest vertical curve (ramp rises from the major highway) or sag vertical curve (ramp falls from the major highway), based on SSD (Table 3) or DSD (Table 4).

The radius of ramp vertical curve (crest or sag) according to driving comfort criterion depends on comfort centrifugal acceleration. The accepted centrifugal acceleration in vertical curves is $0.3 \mathrm{~m} / \mathrm{sec}^{2}$ in order to meet the comfort criterion.

The mechanical expression of this control is presented in Equation (3):

$$
R_{v}=\frac{V_{d}^{2}}{0.3 \cdot 3.6^{2}}=0.2572 \cdot V_{d}^{2}
$$

$R_{r}:$ minimum vertical radius based on driving comfort criterion (m);

$V_{d \cdot}$ design speed (km/hour).

The radius of ramp crest vertical curve according to safety criterion depends 
Table 3. Ramp vertical curve radii minimum design values according to SSD (along the ramp towards an intersection without additive lanes).

\begin{tabular}{|c|c|c|c|c|c|c|c|c|}
\hline \multirow{2}{*}{ Vertical curve type } & \multicolumn{8}{|c|}{ Ramp design speed (km/hour) } \\
\hline & 30 & 40 & 50 & 60 & 70 & 80 & 90 & 100 \\
\hline \multicolumn{9}{|c|}{ Crest vertical curve radii $^{(2)}$} \\
\hline $\begin{array}{l}\text { One lane or two lane directional } \\
\operatorname{ramp}(\text { object height }=0.15 \mathrm{~m})^{(3)}\end{array}$ & $\begin{array}{l}230^{(1)} \\
(160)\end{array}$ & $\begin{array}{l}410^{(1)} \\
(405)\end{array}$ & 760 & 1230 & 2035 & 3320 & 4915 & 4250 \\
\hline \multicolumn{9}{|c|}{ Sag vertical curve $\operatorname{radii}^{(4)}$} \\
\hline $\begin{array}{l}\text { One lane or two lane } \\
\text { directional ramp }\end{array}$ & 230 & 410 & 645 & 930 & 1260 & 1650 & 2085 & 2575 \\
\hline
\end{tabular}

Remarks: ${ }^{(1)}$ The vertical radii for $V_{d}=30,40 \mathrm{~km} /$ hour were derived by comfort criterion for radial vertical acceleration of $0.3 \mathrm{~m} / \mathrm{sec}^{2}\left(R=0.257 \cdot V_{d}^{2}\right)$ as presented by Equation (3). The values in parentheses are computed values derived by the SSD criterion (safety criterion). ${ }^{(2)}$ The vertical radii are suitable for passenger cars for SSD $\leq L$ and for tangents with opposite directions. For SSD $>L$ and tangent with the same directions, relevant formulas (Equation (4), Equation (5)) shall be used [13] [14]. ${ }^{(3)}$ The two-lane, one-way ramp can be categorized as semi-directional ramp ( $\left.V_{d}=80-100 \mathrm{~km} / \mathrm{hour}\right) .{ }^{(4)}$ Sag vertical curves were derived by comfort criterion (Equation (3)) assuming appropriate illumination.

Table 4. Ramp vertical curve minimum design values according to DSD (along the ramp towards a signalized intersection with additive lanes).

\begin{tabular}{|c|c|c|c|c|c|c|c|c|}
\hline \multirow{2}{*}{ Vertical curve type } & \multicolumn{8}{|c|}{ Ramp design speed (km/hour) } \\
\hline & 30 & 40 & 50 & 60 & 70 & 80 & 90 & 100 \\
\hline \multicolumn{9}{|c|}{ Crest vertical curve $\operatorname{radii}^{(2)}$} \\
\hline $\begin{array}{l}\text { One lane or two lane directional } \\
\text { ramp (object height }=0.15 \mathrm{~m})\end{array}$ & 1610 & 3040 & 4570 & 6420 & 9055 & 12,140 & 16,310 & 21,100 \\
\hline \multicolumn{9}{|c|}{ Sag vertical curve radii ${ }^{(3)}$} \\
\hline $\begin{array}{l}\text { One lane or two lane } \\
\text { directional ramp }\end{array}$ & 230 & 410 & 645 & 930 & 1260 & 1650 & 2085 & 2575 \\
\hline
\end{tabular}

Remarks: ${ }^{(1)}$ The vertical radii are suitable for passenger cars for DSD $\leq L$ and for tangents with opposite directions. For DSD > L and tangent with the same directions, relevant formulas shall be used [13] [14]. ${ }^{(2)}$ The two-lane, one-way ramp can be categorized as semi-directional ramp ( $\left.V_{d}=80-100 \mathrm{~km} / \mathrm{hour}\right) \cdot{ }^{\left({ }^{3}\right)} \mathrm{Sag}$ vertical curves were derived by comfort criterion (Equation (3)) assuming appropriate illumination.

on the SSD (or DSD), driver eye height, object height, and the algebraic difference in grade (Equation (4) and Equation (5)). Equation (4) and Equation (5) are different if SSD (or DSD) is smaller or equal to the curve length $(L$ ), or if SSD (or DSD) is larger than the curve length $(L)$ :

$$
\begin{gathered}
R_{v}=\frac{\mathrm{SSD}^{2}}{2 \cdot\left(\sqrt{h_{1}}+\sqrt{h_{2}}\right)^{2}} \text { for } \mathrm{SSD} \leq L \\
R_{v}=\frac{200 \cdot \mathrm{SSD}}{A}-\frac{20000 \cdot\left(\sqrt{h_{1}}+\sqrt{h_{2}}\right)^{2}}{A^{2}} \text { for SSD }>L
\end{gathered}
$$

$R_{V}-$ radius of crest vertical curve (m); 
$A$-algebraic difference in grade (percents);

SSD_stopping sight distance for design (m). DSD can be applied too;

$h_{1}$-design value of driver eye height ( $1.05 \mathrm{~m}$ for passenger cars);

$h_{2}$-design value of the object height: 0.15 meters for ramps towards intersection (for SSD, DSD) or along the major highway towards exit ramp (for DSD); 0.0 meters on entry ramp terminal along acceleration lane (for DSD).

Collisions on ramps and connecting roads generally increase with traffic volume and with decreasing curve radius [1] [4]. Additionally, since upgrade exit ramps have lower collision rates, it is preferable from safety point of view for the connecting road to pass over the freeway or major highway (higher speed road) as presented in the upper part of Figure 2.

\section{Implementing Decision Sight Distance (DSD) on Ramp Terminals}

\subsection{Implementing DSD for Vertical Curve Design on Entry Ramp Terminal along Acceleration Lane}

\subsubsection{Entrance Ramp Terminals: Literature Review}

Implementation of decision sight distance is documented in Canadian geometric design guidelines [1] [2]. At the entrance bull-nose, the driver searches for gaps in traffic along the adjacent lane in order to make a lane change maneuver and merge to the major highway. If the ramp is significantly higher or lower the driver may find it difficult to complete a safe merge. In order to complete a safe merge the driver needs a view of the entire speed change lane at the bull-nose as presented in Figure 3. If the speed change lane (auxiliary lane) alignment includes a crest vertical curve the driver may not have this sight view. A possible solution is to shift the crest vertical curve away from the speed change lane, otherwise, either the speed change lane should be lengthened or the crest vertical curve should be flattened in order to provide DSD to the end of the taper [1]. Typical range of DSD design values for this purpose is presented in Table 6. The Israeli DSD design values are slightly lower than the lower range values.

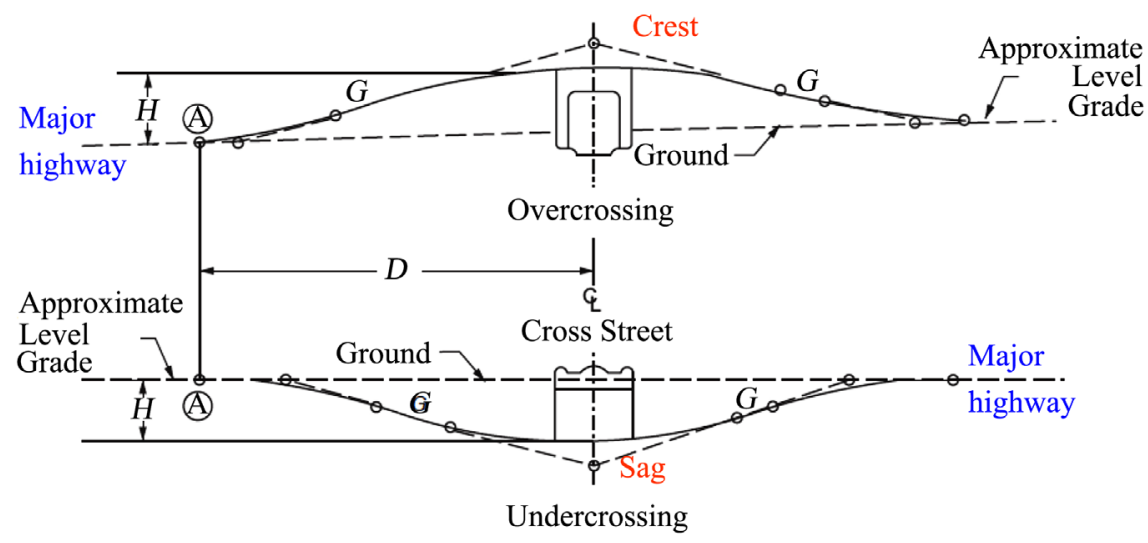

Figure 2. Typical vertical alignment along the ramp (based on AASHTO [9], Fig. 10.8). 


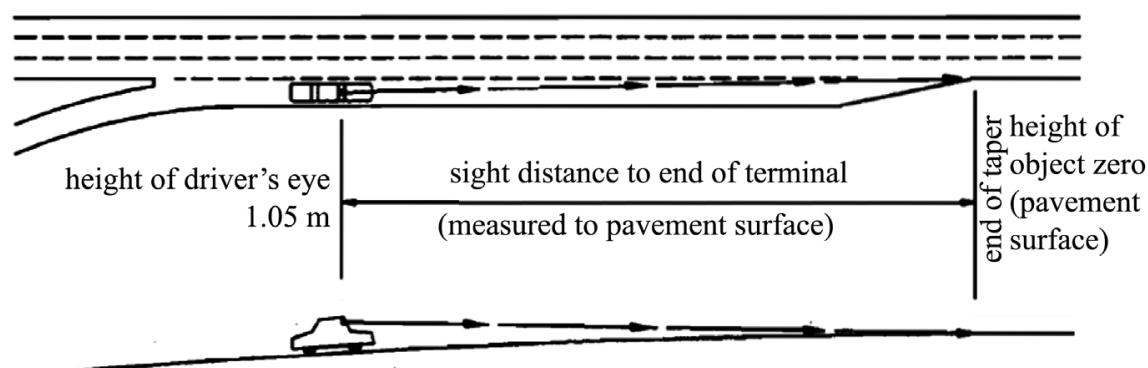

Figure 3. Sight distance at entrance terminals and single lane entry with auxiliary lane: typical examples from Canada and Australia (based on TAC [1], Ontrio [2]).

In Austroads ([3] "interchanges") it is recommended that the distance of travel along a parallel auxiliary lane (acceleration lane) might be 4 seconds of travel by the merging driver (Table 5). An extension might be performed based on either traffic analysis or to guarantee approach sight distance (ASD) to go over a crest vertical curve. These distances proposed by Austroads [3] for entry with auxiliary lanes are significantly lower than DSD.

\subsubsection{Entrance Ramp Terminals: Implementing DSD for Vertical Curve Design (Proposed Implementation)}

In order to enable safe merging to the major highway of the interchange, the driver needs to have a clear sightline along the whole acceleration lane from the entrance ramp zone. If the highway alignment includes a crest vertical curve it has to be designed according to DSD criteria. In Israel, interchanges are mostly illuminated, and therefore a sag vertical curve has to meet comfort criterion but not safety (sight distance) criterion.

DSD should be maintained from the end of the ramp controlling curve (beginning of acceleration lane) until the end of the acceleration lane. The end of the acceleration lane is defined at the point where the lane operational width is $3.60 \mathrm{~m}$ (point $\mathrm{B}$ in Figure 4). The driver, therefore, acknowledges that at this decision point (point B) he/she needs to merge to the major highway. Practically the decision sightline zone can be measured until an operational lane width of $3.0 \mathrm{~m}$ (Figure 4).

At the decision point (B, end of acceleration lane) with object height of $0.0 \mathrm{~m}$ (similar to Australia [3] and Canada [1] [2], the driver reaches his running speed ( $V_{a}$, Table 10-3, [9]) which is lower than the design speed on the major highway of the interchange. Since the speed increases gradually along the acceleration lane, the implementation of running speed has a sufficient safety factor. The decision sightline zone of the entrance ramp terminal is shown in Figure 4.

Table 6 presents the computed DSDs adjusted to the running speed $V_{a}$ at the end of the acceleration lane (point B). In addition, Table 6 includes the minimum radii of crest vertical curve along the acceleration lane (based on DSD safety criterion), and the minimum radii of sag vertical curve along the acceleration lane (based on comfort criterion). If the minimum radius of crest vertical curve is not feasible the acceleration lane should be lengthened. 


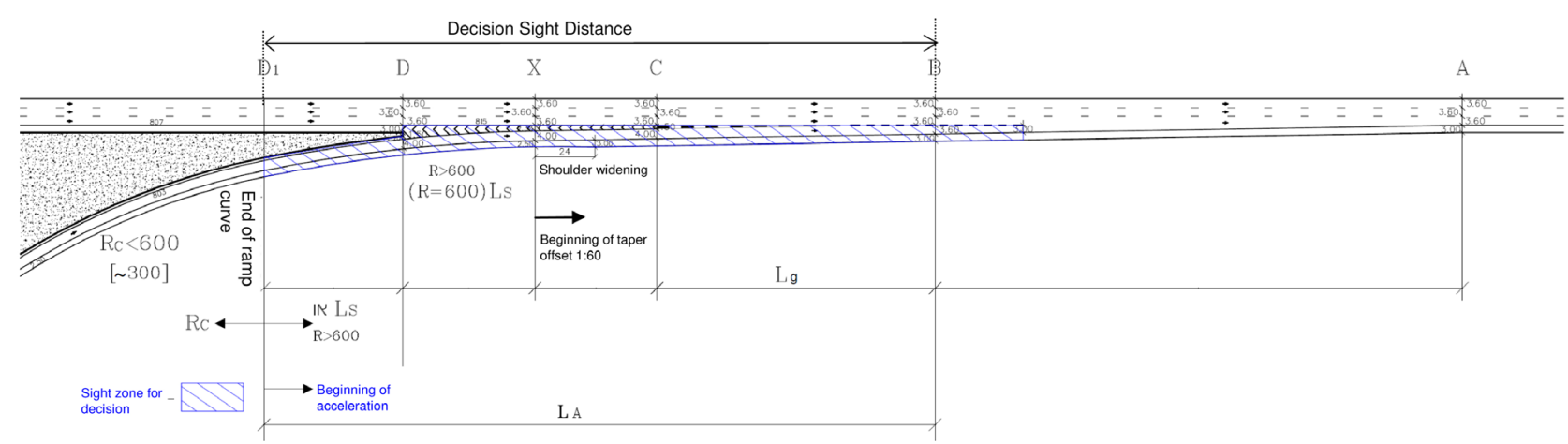

Figure 4. Schematic sight-zone layout of entrance ramp terminal: zone for applying decision sight distance (proposed implementation).

Table 5. Sight distance at entrance terminals and length of parallel lane at entry ramps (based on Ontario [2], Austroads [3]).

\begin{tabular}{cccccccc}
\hline Design speed (km/hour) & 60 & 70 & 80 & 90 & 100 & 110 & 120 \\
\hline $\begin{array}{c}\text { Sight distance to end of } \\
\text { terminal [2] [meter] }\end{array}$ & $170-230$ & $200-270$ & $240-310$ & $270-350$ & $300-390$ & $340-430$ & $370-470$ \\
$\quad$ DSD (Israel) [m] & 160 & 190 & 220 & 255 & 290 & 325 & 360 \\
$\begin{array}{c}4 \text { seconds of travel time: } \\
\text { length of parallel lane } \\
\text { entry ramp [3] [meter] }\end{array}$ & 67 & 78 & 89 & 100 & 111 & 122 & 133 \\
$\begin{array}{c}\text { SSD for ramps (Israel } \\
\text { recommended) [m] }\end{array}$ & 70 & 90 & 115 & 140 & 170 & 200 & 235 \\
\hline
\end{tabular}

Table 6. DSD and vertical curve radii along acceleration lane at entrance ramp terminal zone (recommended).

\begin{tabular}{|c|c|c|c|c|c|}
\hline Design speed $\left(V_{d}\right)$, major highway ( $\mathrm{km} /$ hour $)$ & 80 & 90 & 100 & 110 & 120 \\
\hline Running speed $\left(V_{a}\right)$, end of acceleration lane (km/hour) & 70 & 77 & 85 & 91 & 98 \\
\hline Decision sight distance $(\mathrm{m})$ & 190 & 210 & 235 & 255 & 285 \\
\hline Minimum crest vertical curve radium $(\mathrm{m})^{(1)}$ & 17,190 & 21,000 & 26,300 & 30,970 & 38,980 \\
\hline Minimum sag vertical curve radium (m) & 1260 & 1525 & 1860 & 2130 & 2470 \\
\hline
\end{tabular}

${ }^{(1)}$ The vertical radii are suitable for passenger cars for DSD $\leq L$ and for tangents with opposite directions. For DSD $>L$ and tangent with the same directions, relevant formulas (Equation (4) and Equation (5)) shall be used [13] [14].

\subsection{Implementing DSD for Vertical Curve Design along the Interchanged Highway towards Ramp Terminals}

\subsubsection{Exit Ramp Terminals: Literature Review}

A driver approaching an exit terminal, especially on a freeway is required to make several decisions to complete the maneuver safely. The driver should make suitable adjustments in speed and maneuver into speed change lane (deceleration lane) without conflicts with through traffic. It is important for the driver to see the pavement surface at the bull-nose from a distance longer than SSD i.e. at least equals to DSD [1] [2] as presented in Figure 5(a). A range of design values 


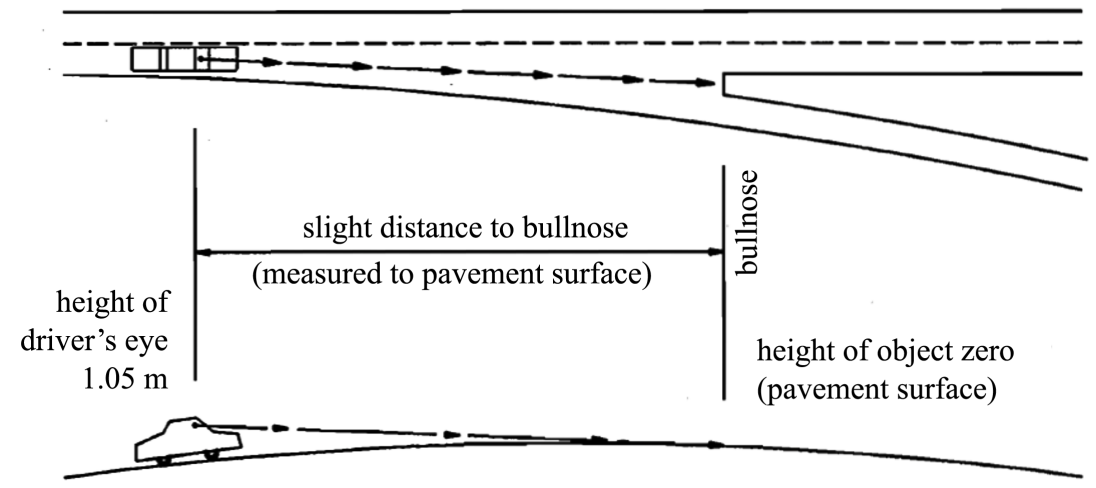

(a)
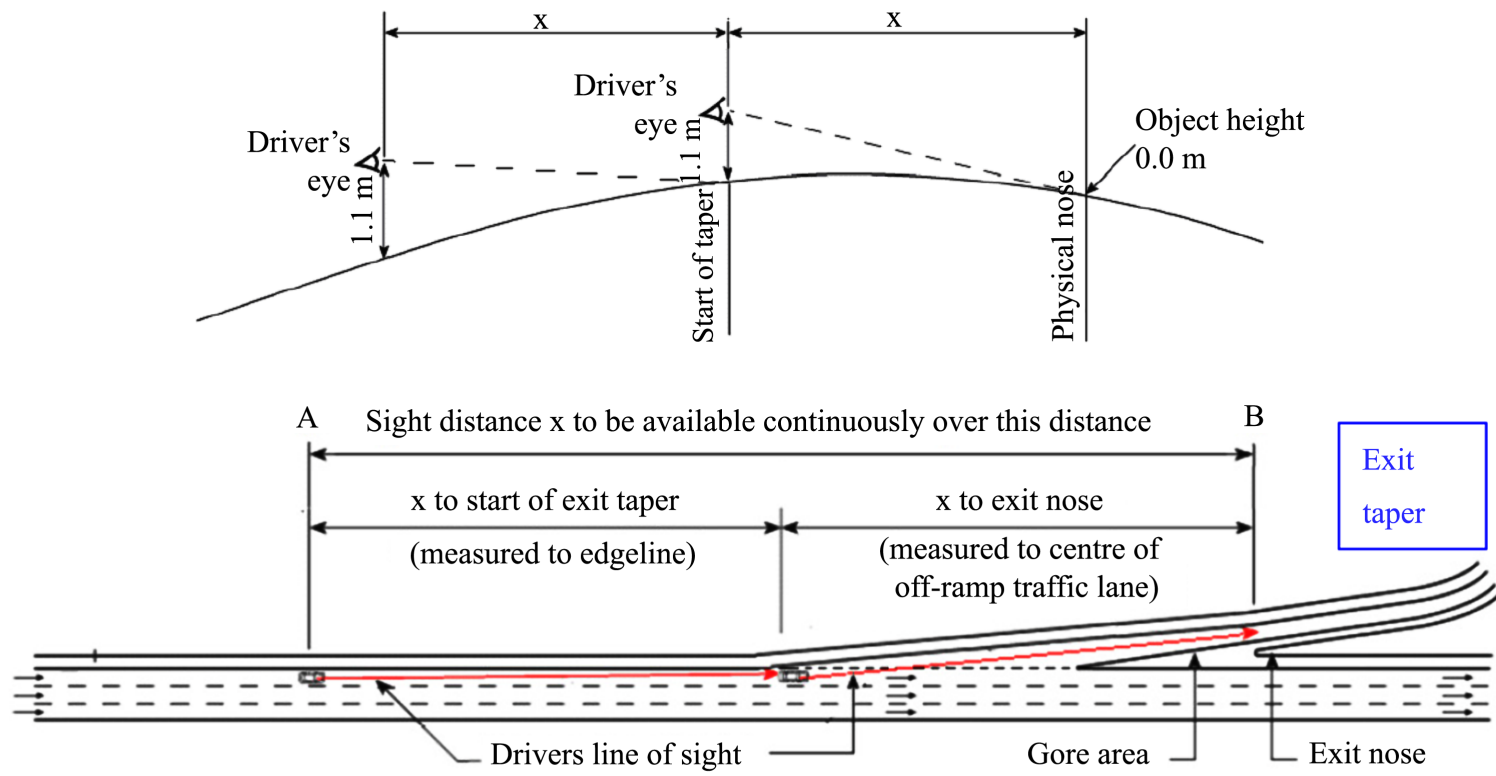

Note: $\mathrm{x}=$ distance travelled in 10 seconds at the operating speed of the major road (measured from $1.1 \mathrm{~m}$ to pavement level)

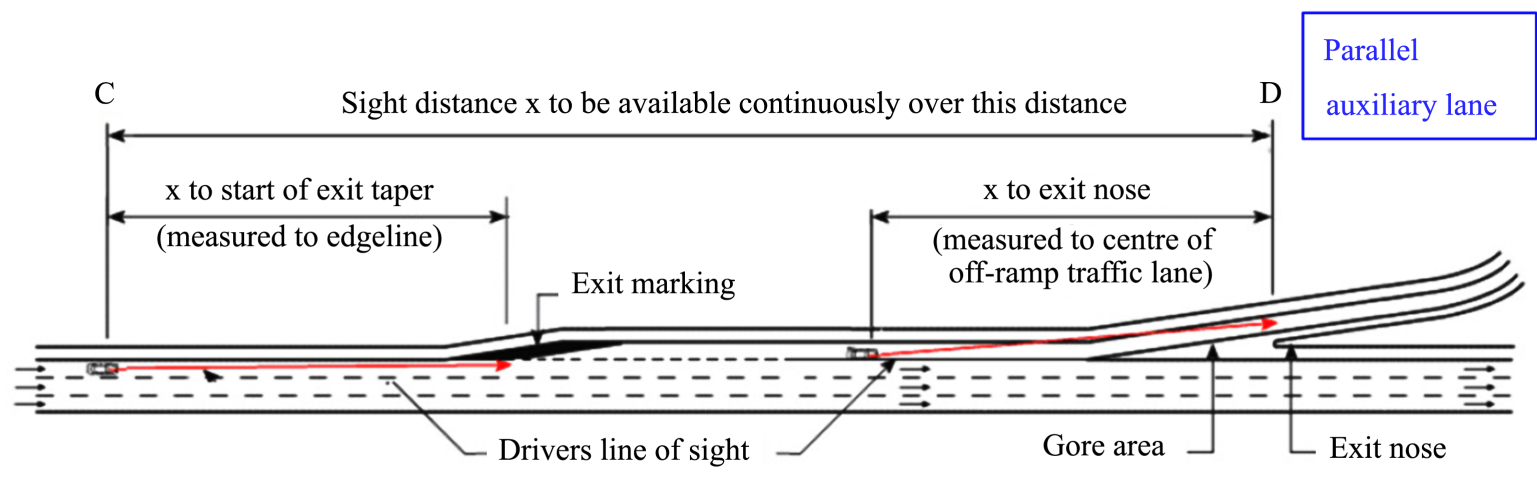

Note: " $x "=$ distance travelled in 7 seconds at the operating speed of the major road (measured from $1.1 \mathrm{~m}$ to pavement level)

(b)

Figure 5. (a) Sight distance at exit ramp terminals: typical example from Canada (based on TAC [1], Ontario [2]); (b) Sight distance requirements at exit ramps with tapered exit and parallel auxiliary lane ramp terminals: typical examples from Australia [3]. 
of Ontario highway design standards [2] is documented in Table 7. The range is identical to the entrance terminals (Table 6) whereas the lower value in the range could be suitable for rural traffic conditions which are less complex than urban traffic conditions.

In Austroads ([3] "interchanges") it is proposed that the driver will search for the start of the exit taper (stage 1) and afterwards for the alignment of the exit lane and the position of exit nose (stage 2). These stages and the required sight distance ( $\mathrm{x}$ ) for either one, are suitable along a tapered lane or an auxiliary lane at exit ramps (Figure 5(b)). These SDs are measured from a driver eye height of $1.1 \mathrm{~m}$ to an object height of pavement surface $(0 \mathrm{~m})$ in order to see the pavement marking at the start of ramp taper (i.e. gore area).

For tapered exit the sight distance required $(x)$ should be equivalent to 10 seconds of travel at design speed. However, the exit prior to a parallel auxiliary lane is more noticeable to the driver, such that 7 seconds of travel time at design speed are sufficient. In both cases (tapered exit or auxiliary lane) the sight distance $x$ should be available continuously along both stages. Obviously, either 7 or 10 seconds of travel time are closer to the Israeli proposed DSD as presented in Table 7.

\subsubsection{Exit Ramp Terminals: Implementing DSD for Vertical Curve Design (Proposed Implementation)}

DSD is the minimum sight distance for designing fully interchanged highway along the ramp terminal influence zones. The implementation of DSD is vital especially towards exit ramps. The design values for DSD are given in Table 2.

The recommended object height to be implemented for DSD (which is essential for calculation crest vertical curve radii) along the multilane interchanged highway prior to ramp terminals and in the interchange influence zone is $0.15 \mathrm{~m}$ (curbstone height of physical nose). This recommended object height is different

Table 7. Sight distance at exit ramp terminals (based on Ontario [2], Austroads [3]).

\begin{tabular}{|c|c|c|c|c|c|c|c|}
\hline $\begin{array}{l}\text { Design speed } \\
\text { (km/hour) }\end{array}$ & 60 & 70 & 80 & 90 & 100 & 110 & 120 \\
\hline $\begin{array}{c}\text { Sight distance at } \\
\text { exit ramp terminals } \\
{[2] \text { [meter] }}\end{array}$ & $170-230$ & $200-270$ & $240-310$ & $270-350$ & $300-390$ & $340-430$ & $370-470$ \\
\hline $\begin{array}{c}7 \text { seconds of travel } \\
\text { time: two } \\
\text { stages-sight distance } \\
\text { (x) for tapered exit } \\
\text { [3] [meter] }\end{array}$ & 117 & 136 & 156 & 175 & 194 & 214 & 233 \\
\hline $\begin{array}{l}10 \text { seconds of travel } \\
\text { time: two stages-sight } \\
\text { distance }(\mathrm{x}) \text { for } \\
\text { parallel auxiliary lane } \\
\text { exit [3] [meter] }\end{array}$ & 167 & 194 & 222 & 250 & 278 & 306 & 333 \\
\hline $\begin{array}{c}\text { DSD (Israel } \\
\text { recommended) }[\mathrm{m}]\end{array}$ & 160 & 190 & 220 & 255 & 290 & 325 & 360 \\
\hline
\end{tabular}


than the recommended object height by Australia [3] and Canada [1] [2] as pavement surface.

DSD can be measured from the gore nose backward along the major interchanged highway. Table 8 presents the DSD, minimum crest vertical curve radii based on safety criterion (DSD), and minimum sag vertical curve radii based on comfort criterion, along the interchanged highway prior to interchange ramp terminals.

This scenario refers to DSD which is adjusted to design speed (Vd) and not to running speed ( $\mathrm{Va}$ ). The assumption that the sag vertical curve is always illuminated in the interchange influence zone is valid here as well, and therefore the sag vertical curve radii were derived by comfort criterion.

The decision sightline zone towards exit ramp along the major highway is shown in Figure 6.

\section{Decision Sight Distance Implementation for a Basic Lane Reduction}

\subsection{Basic Lane Reduction after a Two-Lane Exit Ramp Terminal}

If a significant decrease in traffic volume is expected along the major highway after passing an exit ramp, such that two basic lanes (instead of 3 basic lanes) could accommodate the traffic volume (for at least 2 kilometers), without a deterioration in LOS ( $C$ for a freeway in level terrain, otherwise $\mathrm{D}$ ), then it might be possible to reduce one basic lane. The intention is that after merging in the same

Table 8. DSD and vertical curve radii along the interchanged highway towards ramp terminals (recommended).

\begin{tabular}{cccccc}
\hline Design speed $\left(V_{d}\right)$, major highway $(\mathrm{km} / \mathrm{hour})$ & $\mathbf{8 0}$ & $\mathbf{9 0}$ & $\mathbf{1 0 0}$ & $\mathbf{1 1 0}$ & $\mathbf{1 2 0}$ \\
\hline Decision sight distance $(\mathrm{m})$ & 220 & 255 & 290 & 325 & 360 \\
Minimum crest vertical curve radium $(\mathrm{m}))^{(1)}$ & 12,140 & 16,310 & 21,100 & 26,490 & 32,500 \\
Minimum sag vertical curve radium $(\mathrm{m})$ & 1650 & 2100 & 2575 & 3100 & 3700 \\
\hline
\end{tabular}

${ }^{(1)}$ The vertical radii are suitable for passenger cars for DSD $\leq L$ and for tangents with opposite directions. For DSD > $L$ and tangent with the same directions, relevant formulas (Equation (4), Equation (5)) shall be used [13] [14].

Decision Sight Distance (DSD)

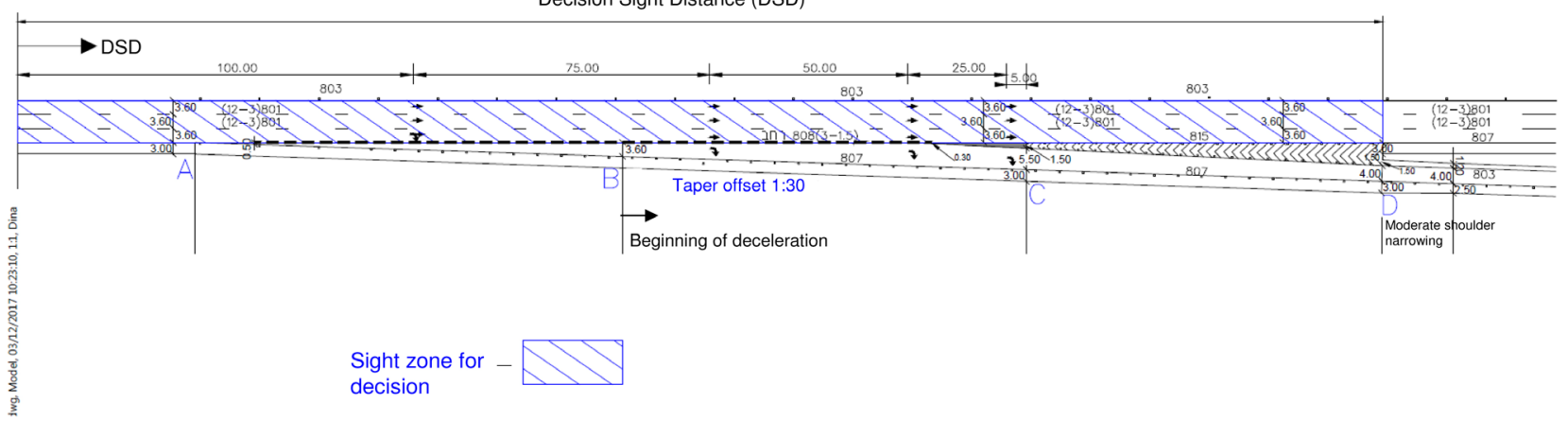

Figure 6. Schematic sight-zone layout towards exit ramp terminal: zone for applying decision sight distance (proposed implementation). 
interchange, the difference between traffic volumes (exit and entrance) will still sustain as a traffic volume of one lane.

In principle the reduction of a basic traffic lane shall be applied after the physical nose as follows:

Decision sight distance (DSD) should be maintained between the edge of the painted nose (point $\mathrm{C}$ ) until the beginning of the taper which directs the basic lane reduction. Figure 7 introduces a DSD of $325 \mathrm{~m}$ for a design speed of 110 $\mathrm{km} /$ hour in the major highway.

A traffic sign for lane reduction (enumerated as 621 in Israel) will be posted 150 meters prior to the beginning of basic lane reduction. For DSD $=325 \mathrm{~m}$ (derived by $V_{d}=110 \mathrm{~km} /$ hour), the traffic sign is located 85 meters after the physical nose.

The number of arrows shown in traffic sign number 621, will be identical to the number of lanes downstream, i.e. the number of legs will be equal to the number of lanes prior to lane reduction.

The taper for lane reduction will have an offset of 1:100 (length of 360 meters). The "reduced" lane is the rightmost lane.

\subsection{Basic Lane Reduction Implemented Prior to a Two-Lane Exit Ramp Terminal}

A reduction of a basic lane prior to a two-lane exit ramp terminal is not recommended on freeways or interchanged highway. The logic is a bottleneck potential along the influence zone of basic lane reduction (approximated length of 360 $\mathrm{m})$. This influence zone of basic lane reduction should be suitable for completing successfully the weaving maneuver which has two simultaneous traffic actions: 1) passing from the right lane to one of the left lanes for the vehicles driving along the major highway; 2) passing from the left lanes to the reduced basic lane (practically the exit lane to the right) for drivers exiting through the exit ramp terminal. DSD in such a scenario will be managed by directional and message signs which are posted prior to the beginning of basic lane reduction (Figure 8). This geometric design solution could be implemented in constrained situations, practically intermediate stage of construction or right of way limitations which are typical in Israel.

\section{Summary and Conclusions}

The paper presents safety background and sight distance criteria in the geometric design of interchanges and junctions, specifically, implementation of stopping sight distance (SSD) and decision sight distance (DSD). These sight distance (SD) types are utilized in the design of ramps, ramp terminals (along acceleration lane and along the major highway towards exit ramps), and in the implementation of basic lane reduction before and after two-lane exit ramp terminal.

Overall, interchanges present the vehicle driver with a complex set of decisions which require quick evaluation and action. Reduction of driver's stress at 


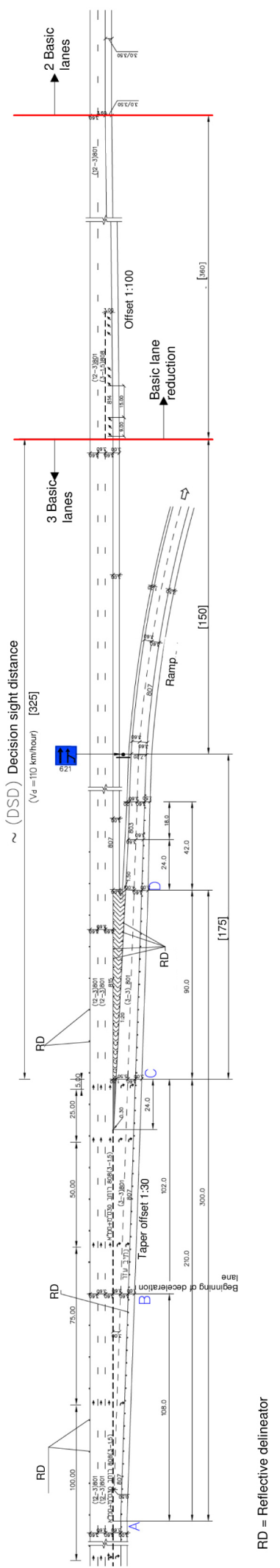

Figure 7. Basic lane reduction implemented after a two-lane exit ramp terminal ( $V_{d}=100$ $\mathrm{km}$ /hour). 


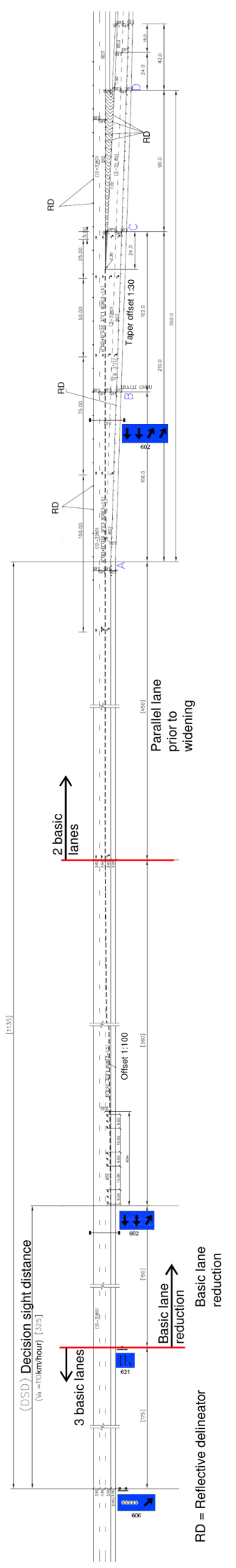

Figure 8. Basic lane reduction prior to a two-lane exit ramp terminal. 
interchanges can be performed by: keeping the alignment simple and direct, maintaining design consistency, providing sufficient sight distance (specifically decision sight distance), and using resulting minimum design criteria for the geometric components. These design criteria might alleviate safety by reducing conflicts and confusion to the driver, and expanding the decision points. The implementation of decision sight distance in two lane exit ramps prior to basic lane reduction and utilization of message traffic signing might improve driver uncertainty and contribute to crash mitigation.

Still, exit ramps along inter-urban highways encounter higher crash rates than entrance ramps. It appears that all exits (two lane exit terminals as well as one lane exit terminals) should be suitably signed with sufficient coherent and visible information for the drivers. This might reduce the risk of crashes while performing and completing the exit maneuver.

A decisive application of DSD is traffic control and Intelligent Transportation Systems. The DSD length could be utilized to examine the necessity of advance warning message sign. The sign might assist the driver to reduce the pre-maneuver time component of the DSD, specifically the detection and recognition, and reduce the probability of colliding the hazard.

An initiative decision of the driver, while the driver expects a hazard or an intersection at the ramp edge (with intersecting minor highway), might result in a shorter pre-maneuver time and therefore, a shorter decision sight distance. Every project should be treated according to its interchanges' characteristics and accompanied highway system.

If the sighting distance is too short, then warning signs could inform the driver which maneuver or maneuver alternatives should be considered in order to escape from the obstruction.

The use of collector distributor (CD) roads might encourage speed reduction (by an intermediate speed facility) and therefore provide a longer pre-maneuver time and maneuver time especially for more complex exits which necessitate series of decisions.

\section{Conflicts of Interest}

The author declares no conflicts of interest regarding the publication of this paper.

\section{References}

[1] TAC. Transportation Association of Canada (2017) Geometric Design Guide for Canadian Roads.

[2] Geometric Design Standards for Ontario Highways (1985) Chapter F: Interchanges. Ontario Ministry of Transportation. Surveys \& Design Office, Downsview, Ontario, Canada.

[3] Austroads (2009) Guide to Road Design, Part 3: Geometric Design, AGRD03/09. Austroads, Sydney, NSW.

[4] Twomey, J.M., Heckman, M.L. and Hayward, J.C. (1992) Safety Effectiveness of 
Highway Design Features. Volume IV. Interchanges. FHWA-RD-91-047. Federal Highway Administration.

[5] Torbic, D.J., Harwood, D.K., Gilmore, D.K., Richard, K.R. and Bared, J.G. (2009) Safety Analysis of Interchanges. Transportation Research Record 2092, 39-47.

[6] Alexander, G.J. and Lunenfeld, H. (1990) A Users' Guide to Positive Guidance. 3rd Edition, Federal Highway Administration, USA.

[7] Bassan, S. (2011) Decision Sight Distance Review and Evaluation. Traffic Engineering and Control, 52, 23-26.

[8] McGee, H.W. (1979) Decision Sight Distance for Highway Design and Traffic Control Requirements. Transportation Research Record 736, 11-13.

[9] American Association of State Highway and Transportation Officials (AASHTO) (2011) A Policy on Geometric Design of Highways and Streets. 6th Edition, Washington DC.

[10] American Association of State Highway and Transportation Officials (AASHTO) (2018) A Policy of Geometric Design of Highways and Streets. 7th Edition, Washington DC.

[11] Austroads (2003) Rural Road Design. A Guide to the Geometric Design of Rural Roads. Austroads, Sydney, NSW.

[12] Bassan, S. (2019) Review and Design Policy of Sight Distance Types for the Interurban Highway System. Proceedings of the Institution of Civil Engineers. Transport, 172, 274-288. https://doi.org/10.1680/jtran.17.00075

[13] Olson, P.L., Cleveland, D.E., Fancher, P.S. and Schneider, L.W. (1984) Parameters Affecting Stopping Sight Distance. Final Report. The University of Michigan. Transport Research Institute, Ann Arbor, Michigan. Prepared for: National Cooperative Highway Research Program. Transportation Research Board. National Research Council. http://mirlyn.lib.umich.edu/Record/004951575

[14] Bassan, S. (2017) Vertical Curve Design Insights of Road Tunnels versus Highways. Journal of Transportation Safety \& Security, 9, 319-346. https://doi.org/10.1080/19439962.2016.1206049 